Volume 5, Issue 2, 2019, p. 35-36

\title{
Adhesive small bowel obstruction: where do we stand now?
}

\author{
Barbara Leone ${ }^{1,2}$, Giuseppe Cipollone ${ }^{2}$, Decio Di Nuzzo², Massimo Ippoliti², Mirko Barone ${ }^{2 *}$, Felice \\ Mucilli2 $^{2}$
}

'School of Medicine, University “G.d'Annunzio" Chieti - Pescara, via dei Vestini n. 100, 66100 Chieti, Italy

2Department of General and Thoracic Surgery, University Hospital of Chieti, via dei Vestini n. 1, 66100 Chieti, Italy

\begin{abstract}
Dear Editor
Adhesive small bowel obstruction (ASBO) is one of the most frequently encountered disorders in Emergency Surgery Departments worldwide without negligible hospital admission rates and social costs $(1,2)$. Notwithstanding significant improvements in techniques and materials, intra-abdominal adhesions following abdominal surgery still represent a major unsolved and debated issue harbouring challenges regarding diagnosis, pathogenesis, management and prevention. In this setting, the cornerstone lies on a proper nosological classification with a subsequent diagnostic dilemma in distinguishing ASBO from other causes of obstruction and in an early identification of emergency surgery cases. Moreover, conflicting results raise more questions. As known, fibrous bands occur in up to $93 \%$ of patients undergoing abdominal procedures with a considerable rate of future complications, resulting in morbidity, mortality and a $30 \%$ re-admission rate at 4 years after surgery $(3,4)$. For these reasons, recent studies and systematic reviews have pointed out the "prevention" by considering the socioeconomic burden of adhesiolyses (5-7). But, where are we standing now? Should we reconsider our mind? Do minimally invasive surgery or pharmacological barriers really reduce ASBO incidence? Does ASBO relate to the age and chronicity of diseases? Are we witnessing an epidemiological change? In reality, we are still relegated to uncertainty and to conflicting experiences, despite
\end{abstract}

\footnotetext{
Received: 5 June 2018; Accepted: 15 September 2018;

Published online: 25 September 2018

*Corresponding author: Mirko Barone, MD; Department of General and Thoracic Surgery, University Hospital of Chieti, via dei Vestini n. 1, 66100 Chieti, Italy. Tel: +390871 358289, Fax: +39 0871 358220,

Email:mir87mb@libero.it

Competing interests: None.

Funding information: There is none to be declared

Citation: Leone B, Cipollone G, Di Nuzzo D, Ippoliti M, Barone M, Mucilli F. Adhesive small bowel obstruction: where do we stand now? Journal of Emergency Practice and Trauma 2019; 5(2): 35-36. doi: 10.15171/ jept.2018.12
}

the presence of established diagnostic pathways and guidelines. Nowadays, it is essential to contextualise bowel obstructive syndromes to the increase of the general population's average age and its related co-morbidities.

For these reasons, an invasive treatment should be quick, minimally invasive and rather decisive. But, is less better? Nakamura et al (8), in a retrospective study involving $123 \mathrm{ASBO}$ patients and comparing open to laparoscopic approach, reported that open surgery was the only independent risk factor for the recurrence of small bowel obstruction (odds ratio [OR]: 5.621; $P=0.015)$ at multivariate analysis. Similarly, Aquina et al (9), investigating the effects of a minimally invasive strategy for colorectal resections in adhesions formation, confirmed that laparotomy was associated with a higher risk of both SBO (hazard ratio [HR]: 1.14, 95\% CI: 1.031.26) and operation for ASBO (HR: 1.12, 95\% CI: 0.941.32). This effect was even greater when characterizing laparoscopic-to-open conversions as an open approach (SBO: HR 1.34, 95\% CI: 1.20-1.49; SBO operation: HR $1.35,95 \%$ CI: $1.12-1.63)$.

If on the one hand, a general trend towards minimally invasive approaches appears clear, the same can be also asserted about measures of prevention such as pharmacological agents and anti-adhesive barriers (7). Encouraging results were published on the POPA study (6) and on the ADEPT trial (10) concerning the intraperitoneal injection of icodextrin $4 \%$ during surgery with a significant reduction both in early and morbidity rates with a proven pharmacological safety, confirming the evidences of a wider Cochrane Systematic Review (11). So, why do intestinal occlusions still remain a tedious open problem? What justifies the increasing rates of relapse? In our opinion, causes are to be found in several factors. First of all, we have to face with usability and technology diffusion issues. These aspects, intimately linked to social and healthcare costs, make such strategies inaccessible to most of patients, especially in developing countries. However, subordinating health to expenditures 
and to the lack of implementing instruments would lead to an irremediable gap and to an unequal treatment. A further aspect is the lack of education. Too often ASBO patients are left to arbitrary decisions based on experience and not on current guidelines, affecting both outcome and mortality. A normalization and a revision in "convictions" currently urge more than ever.

\section{Ethical issues}

Not applicable.

\section{Authors' contributions}

Manuscript design and Literature research: Barbara Leone; Results discussion: All Authors; Manuscript writing: All Authors.

\section{References}

1. ten Broek RPG, Issa Y, van Santbrink EJP, Bouvy ND, Kruitwagen RFPM, Jeekel J, et al. Burden of adhesions in abdominal and pelvic surgery: systematic review and metanalysis. BMJ 2013; 347: f5588. doi: 10.1136/bmj.f5588.

2. Loftus T, Moore F, VanZant E, Bala T, Brakenridge S, Croft $\mathrm{C}$, et al. A protocol for the management of adhesive small bowel obstruction. J Trauma Acute Care Surg 2015; 78(1): 13-9; discussion 9-21. doi: 10.1097/ta.0000000000000491.

3. Parker MC, Ellis H, Moran BJ, Thompson JN, Wilson MS, Menzies D, et al. Postoperative adhesions: ten-year followup of 12,584 patients undergoing lower abdominal surgery. Dis Colon Rectum 2001; 44(6): 822-29; discussion 9-30.

4. Ellis H, Moran BJ, Thompson JN, Parker MC, Wilson MS, Menzies D, et al. Adhesion-related hospital readmissions after abdominal and pelvic surgery: a retrospective cohort study. Lancet 1999; 353(9163): 1476-80. doi: 10.1016/ s0140-6736(98)09337-4.

5. ten Broek RP, Strik C, Issa Y, Bleichrodt RP, van Goor
H. Adhesiolysis-related morbidity in abdominal surgery. Ann Surg 2013; 258(1): 98-106. doi: 10.1097/ SLA.0b013e31826f4969.

6. Catena F, Ansaloni L, Di Saverio S, Pinna AD. P.O.P.A. study: prevention of postoperative abdominal adhesions by icodextrin $4 \%$ solution after laparotomy for adhesive small bowel obstruction. A prospective randomized controlled trial. J Gastrointest Surg 2012; 16(2): 382-8. doi: 10.1007/ s11605-011-1736-y.

7. Ten Broek RPG, Stommel MWJ, Strik C, van Laarhoven CJHM, Keus F, van Goor H. Benefits and harms of adhesion barriers for abdominal surgery: a systematic review and meta-analysis. Lancet 2014; 383(9911): 48-59. doi: 10.1016/ s0140-6736(13)61687-6.

8. Nakamura T, Sato T, Naito M, Ogura N, Yamanashi T, Miura $\mathrm{H}$, et al. Laparoscopic Surgery is Useful for Preventing Recurrence of Small Bowel Obstruction After Surgery for Postoperative Small Bowel Obstruction. Surg Laparosc Endosc Percutan Tech 2016; 26(1): e1-4. doi: 10.1097/ sle.0000000000000238.

9. Aquina CT, Probst CP, Becerra AZ, Iannuzzi JC, Hensley BJ, Noyes K, et al. Missed Opportunity: Laparoscopic Colorectal Resection Is Associated With Lower Incidence of Small Bowel Obstruction Compared to an Open Approach. Ann Surg 2016; 264(1): 127-34. doi: 10.1097/ sla.0000000000001389.

10. Sakari T, Sjodahl R, Pahlman L, Karlbom U. Role of icodextrin in the prevention of small bowel obstruction. Safety randomized patients control of the first 300 in the ADEPT trial. Colorectal Dis 2016; 18(3): 295-300. doi: 10.1111/codi.13095.

11. Ahmad G, Mackie FL, Iles DA, O'Flynn H, Dias S, Metwally $\mathrm{M}$, et al. Fluid and pharmacological agents for adhesion prevention after gynaecological surgery. Cochrane Database Syst Rev 2014; (7): Cd001298. doi: 10.1002/14651858. CD001298.pub4. 